\title{
ARTICLE OPEN \\ Pathways to diagnosis of non-small cell lung cancer: a descriptive cohort study
}

Stuart Purdie ${ }^{1}$, Nicola Creighton (D) ${ }^{1}$, Kahren Maree White ${ }^{1}$, Deborah Baker ${ }^{1}$, Dan Ewald ${ }^{2,3}$, Chee Khoon Lee ${ }^{4,5}$, Alison Lyon ${ }^{6}$, Johnathan Man ${ }^{7}$, David Michail ${ }^{7}$, Alexis Andrew Miller ${ }^{8,9}$, Lawrence Tan ${ }^{6}$, David Currow ${ }^{1}$ and Jane M. Young ${ }^{10,11}$

Little has been published on the diagnostic and referral pathway for lung cancer in Australia. This study set out to quantify general practitioner (GP) and lung specialist attendance and diagnostic imaging in the lead-up to a diagnosis of non-small cell lung cancer (NSCLC) and identify common pathways to diagnosis in New South Wales (NSW), Australia. We used linked health data for participants of the 45 and Up Study (a NSW population-based cohort study) diagnosed with NSCLC between 2006 and 2012 . Our main outcome measures were GP and specialist attendances, X-rays and computed tomography (CT) scans of the chest and lung cancer-related hospital admissions. Among our study cohort $(N=894), 60 \%(n=536)$ had $\geq 4 \mathrm{GP}$ attendances in the 3 months prior to diagnosis of NSCLC, $56 \%(n=505)$ had GP-ordered imaging (chest X-ray or CT scan), 39\% $(N=349)$ attended a respiratory physician and $11 \%(N=102)$ attended a cardiothoracic surgeon. The two most common pathways to diagnosis, accounting for one in three people, included GP and lung specialist (respiratory physician or cardiothoracic surgeon) involvement. Overall, $25 \%$ of people $(n=223)$ had an emergency hospital admission. For $14 \%$ of people $(N=129)$, an emergency hospital admission was the only event identified on the pathway to diagnosis. We found little effect of remoteness of residence on access to services. This study identified a substantial proportion of people with NSCLC being diagnosed in an emergency setting. Further research is needed to establish whether there were barriers to the timely diagnosis of these cases.

npj Primary Care Respiratory Medicine (2019)29:2; https://doi.org/10.1038/s41533-018-0113-7

\section{INTRODUCTION}

Lung cancer kills more people than any other cancer in Australia. ${ }^{1}$ Five-year survival has improved since the 1980s but remains poor (16\% for 2009-2013). ${ }^{1}$ Evidence-based treatment is critical to further improve outcomes for people with lung cancer. Numerous studies examining treatment pathways for people with lung cancer in Australia have identified gaps in optimal treatment delivery. ${ }^{2-8}$ While there are studies that have focussed on the pathway from the point of specialist referral and treatment planning onwards ${ }^{4-9}$, there are fewer studies on the primary care, diagnostic and referral pathways to the point of specialist contact. $^{10,11}$ To our knowledge, no Australian study has examined the use of diagnostic imaging and specialist referral in the lead-up to lung cancer diagnosis.

Although lung cancer is one of the most common cancers in Australia (43 new cases per 100,000 people per year ${ }^{1}$ ), it will be encountered rarely by an individual general practitioner (GP) and diagnosis can be challenging. The early symptoms of lung cancer, such as weight loss, a new or changing cough, shortness of breath and chest pain, are often non-specific. The absence of symptoms for many people until disease is advanced makes early diagnosis challenging. Haemoptysis, a "red flag" symptom, only occurs in around one fifth of people presenting with lung cancer. ${ }^{12,13}$ Most people require imaging beyond a simple chest X-ray to confirm the diagnosis of lung cancer. ${ }^{14}$
People with suspected lung cancer should be referred for specialist assessment, including obtaining a tissue diagnosis and oversight by a multi-disciplinary team charged with developing a consensus-based treatment plan. ${ }^{14,15}$ New South Wales (NSW) studies found that a lung cancer multidisciplinary team (MDT) increased the use of treatment, ${ }^{16}$ while reduced access to specialist care may account for poorer outcomes for people in rural settings. ${ }^{3,17}$

In this study, we aimed to quantify the use and pattern of GP attendances, specialist attendances and diagnostic imaging in a community/outpatient setting in the lead-up to diagnosis and identify common diagnostic pathways of NSW residents with nonsmall cell lung cancer (NSCLC). A secondary aim was to measure health service use by remoteness of residence.

\section{RESULTS}

A total of 1235 people were diagnosed with NSCLC between enrolment into the 45 and Up Study (February 2006) and 31 December 2012. There were 894 people in the study cohort (Table 1) after excluding cases notified only by death certificate $(n=34)$, people with another cancer case diagnosed between 2000 and $2012(n=251)$ and cases with an estimated diagnosis date $(n=56)$.

\footnotetext{
${ }^{1}$ Cancer Institute NSW, Sydney, NSW, Australia; ${ }^{2}$ North Coast Primary Health Network, Ballina, NSW, Australia; ${ }^{3}$ University Centre for Rural Health, Lismore, NSW, Australia; ${ }^{4}$ Cancer Care Centre, St George Hospital, Sydney, NSW, Australia; ${ }^{5}$ NHMRC Clinical Trials Centre, University of Sydney, Sydney, NSW, Australia; ${ }^{6}$ School of Medicine, Western Sydney University, Sydney, NSW, Australia; ${ }^{7}$ Crown Princess Mary Cancer Centre, Westmead Hospital, Sydney, NSW, Australia; ${ }^{8}$ Illawarra Cancer Care Centre, Wollongong Hospital,

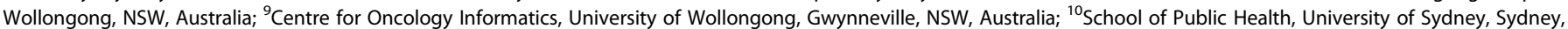
NSW, Australia and ${ }^{11}$ Surgical Outcomes Research Centre, Sydney Local Health District, Sydney, NSW, Australia

Correspondence: Nicola Creighton (Nicola.Creighton@health.nsw.gov.au)
}

Received: 24 May 2018 Accepted: 6 December 2018

Published online: 08 February 2019 
Table 1. Demographic and tumour characteristics of study participants with non-small cell lung cancer

\begin{tabular}{|c|c|c|}
\hline Characteristic & $n$ & $\%(N=894)$ \\
\hline \multicolumn{3}{|l|}{ Age at diagnosis, years } \\
\hline $45-64$ & 195 & 21.8 \\
\hline $65-79$ & 453 & 50.7 \\
\hline $80+$ & 246 & 27.5 \\
\hline \multicolumn{3}{|l|}{ Sex } \\
\hline Female & 385 & 43.1 \\
\hline Male & 509 & 56.9 \\
\hline \multicolumn{3}{|l|}{ Remoteness of residence } \\
\hline Major cities & 477 & 53.4 \\
\hline Inner regional & 314 & 35.1 \\
\hline Outer regional/remote & 103 & 11.5 \\
\hline \multicolumn{3}{|l|}{ Area-based socioeconomic status } \\
\hline Least disadvantaged quintile & 137 & 15.3 \\
\hline Quintile 2 & 147 & 16.4 \\
\hline Quintile 3 & 176 & 19.7 \\
\hline Quintile 4 & 256 & 28.6 \\
\hline Most disadvantaged quintile & 178 & 19.9 \\
\hline \multicolumn{3}{|l|}{ Smoking status at enrolment ${ }^{a}$} \\
\hline Current smoker & 217 & 24.3 \\
\hline Ex-smoker & 524 & 58.6 \\
\hline Never smoked regularly & 150 & 16.8 \\
\hline \multicolumn{3}{|l|}{ Extent of disease at diagnosis } \\
\hline Localised & 173 & 19.4 \\
\hline Regional & 204 & 22.8 \\
\hline Distant & 417 & 46.6 \\
\hline Unknown & 100 & 11.2 \\
\hline \multicolumn{3}{|l|}{ Best method of diagnosis } \\
\hline Histopathologically verified & 642 & 71.8 \\
\hline Cytology & 169 & 18.9 \\
\hline Clinical/imaging/biochemistry & 83 & 9.3 \\
\hline \multicolumn{3}{|l|}{ Charlson comorbidity score } \\
\hline 0 & 682 & 76.3 \\
\hline 1 & 130 & 14.5 \\
\hline$\geq 2$ & 82 & 9.2 \\
\hline
\end{tabular}

Use of health services in the lead-up to diagnosis

The rate of GP attendance was $<1$ per person 15 months prior to NSCLC diagnosis, increasing gradually from 6 months prior to diagnosis (Fig. 1) before reaching a peak of just under two per person in the month of diagnosis. Rapid increases in the number of chest X-rays, chest computed tomography (CT) scans and lung specialist attendances in a community setting coincided with the rapid increase in GP attendances from 2 months before diagnosis.

In the 3 months to diagnosis, $93 \%$ of people attended a GP, with $60 \%$ attending $\geq 4$ times; $39 \%$ attended a respiratory physician; $11 \%$ attended a cardiothoracic surgeon; while $<10 \%$ attended a medical or radiation oncologist, general physician or general surgeon (Table 2). Just under half $(46 \%)$ of people had a diagnostic procedure (bronchoscopy or biopsy) recorded. Just over half had a chest X-ray (53\%) or chest CT scan (59\%) in a community setting, with most imaging ordered by a GP. Around $40 \%$ of people had both a chest X-ray and CT scan and almost a third of people did not have a record of either (Table 3 ). We could

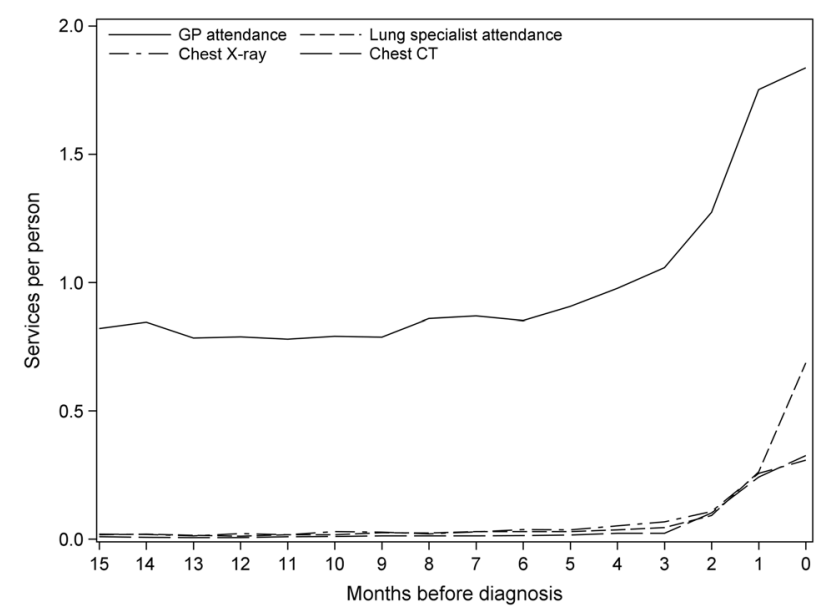

Fig. 1 GP attendances, lung specialist attendances, chest X-rays and chest CT scans per person in the 15 months before non-small cell lung cancer diagnosis

not determine how many people saw a lung specialist or had chest imaging during an inpatient admission.

Although there were some differences in distribution of number of GP attendances, the median and interquartile range of GP attendances were the same for all remoteness areas (median: 4, interquartile range: 3-6). Specialist attendances were similar across remoteness areas. Respiratory physician attendance was not substantially higher $\left(x^{2}(2)=1.98, P=0.37\right)$ for those living in major cities (41\%), compared with inner regional (37\%) and outer regional/remote areas (35\%).

\section{Pathways to diagnosis}

The two most common pathways to diagnosis, accounting for $30 \%$ of people, included GP-ordered imaging and lung specialist attendance without an emergency hospital admission (Table 4). Pathways including a lung specialist attendance $(n=405,45 \%)$ had a higher proportion of cases with a histopathologically confirmed diagnosis $\left(77 \%\right.$ vs $\left.67 \%, X^{2}(1)=11.0, P \leq 0.001\right)$, a lower proportion of emergency hospital admissions ( $8 \%$ vs $39 \%, X^{2}(1)=$ 111.6, $P<0.001)$ and a lower proportion of lung cancers with distant spread $\left(31 \%\right.$ vs $\left.59 \%, X^{2}(1)=69.5, P<0.001\right)$.

The third most common pathway $(n=129,14 \%)$ was an emergency hospital admission without GP-ordered imaging or lung specialist attendance in the community. Overall, 223 people (25\%) had an emergency hospital admission. People with an emergency admission had a higher proportion of cancers with distant spread $\left(67 \%\right.$ vs $\left.40 \%, X^{2}(1)=50.8, P<0.001\right)$ and a lower proportion without comorbidity $\left(69 \%\right.$ vs $79 \%, X^{2}(1)=8.6, P<$ 0.014).

Of the $11 \%(n=96)$ of people with no events of interest (pathway 5), 15 (16\%) saw a general physician or surgeon and 11 (12\%) saw a medical or radiation oncologist.

\section{DISCUSSION}

The use of community-based health services associated with lung cancer diagnosis increased rapidly in the 2 months before diagnosis of NSCLC. The key role that GPs play in the diagnosis of lung cancer is highlighted by the findings that in the 3 months to diagnosis $93 \%$ attended a GP with $60 \%$ of people attending a GP at least four times and a similar proportion having GP-ordered thoracic imaging.

The two most common pathways to diagnosis of NSCLC, accounting for one in three people diagnosed, involved GP and lung specialists in the community without an emergency hospital 


\begin{tabular}{|c|c|c|c|c|c|c|c|c|c|c|}
\hline \multirow[t]{2}{*}{ Service } & \multirow[t]{2}{*}{ Number of services } & \multicolumn{2}{|c|}{$\begin{array}{l}\text { Major cities } \\
(N=477)\end{array}$} & \multicolumn{2}{|c|}{$\begin{array}{l}\text { Inner } \\
\text { regional } \\
(N=314)\end{array}$} & \multicolumn{2}{|c|}{$\begin{array}{l}\text { Outer } \\
\text { regional/ } \\
\text { remote } \\
(N=103)\end{array}$} & \multicolumn{2}{|c|}{$\begin{array}{l}\text { Total } \\
(N=894)\end{array}$} & \multirow[t]{2}{*}{$x^{2}$ test } \\
\hline & & $n$ & $\%$ & $n$ & $\%$ & $n$ & $\%$ & $n$ & $\%$ & \\
\hline \multirow[t]{3}{*}{ GP attendance } & 0 & 35 & 7.3 & 17 & 5.4 & 7 & 6.8 & 59 & 6.6 & $X^{2}(8)=15.66, P=0.05$ \\
\hline & $7-9$ & 79 & 16.6 & 45 & 14.3 & 6 & 5.8 & 130 & 14.5 & \\
\hline & $>9$ & 37 & 7.8 & 17 & 5.4 & 8 & 7.8 & 62 & 6.9 & \\
\hline Respiratory physician attendance & $\geq 1$ & 196 & 41.1 & 117 & 37.3 & 36 & 35.0 & 349 & 39.0 & $X^{2}(2)=1.98, P=0.37$ \\
\hline Cardiothoracic surgeon attendance & $\geq 1$ & 46 & 9.6 & 44 & 14.0 & 12 & 11.7 & 102 & 11.4 & $X^{2}(2)=3.58, P=0.17$ \\
\hline Medical oncologist attendance & $\geq 1$ & 24 & 5.0 & 14 & 4.5 & 7 & 6.8 & 45 & 5.0 & $X^{2}(2)=0.89, P=0.64$ \\
\hline Radiation oncologist attendance & $\geq 1$ & 32 & 6.7 & 20 & 6.4 & 9 & 8.7 & 61 & 6.8 & $x^{2}(2)=0.71, P=0.70$ \\
\hline GP ordered ${ }^{\mathrm{a}}$ & $\geq 1$ & 196 & 41.1 & 150 & 47.8 & 38 & 36.9 & 384 & 43.0 & $x^{2}(2)=5.19, P=0.07$ \\
\hline Specialist ordered ${ }^{\mathrm{a}}$ & $\geq 1$ & 74 & 15.5 & 33 & 10.5 & 12 & 11.7 & 119 & 13.3 & $X^{2}(2)=4.39, P=0.11$ \\
\hline CT scan (outpatient) & $\geq 1$ & 280 & 58.7 & 189 & 60.2 & 62 & 60.2 & 531 & 59.4 & $x^{2}(2)=0.21, P=0.90$ \\
\hline GP ordered ${ }^{a}$ & $\geq 1$ & 200 & 41.9 & 148 & 47.1 & 48 & 46.6 & 396 & 44.3 & $x^{2}(2)=2.33, P=0.31$ \\
\hline Specialist ordered ${ }^{\mathrm{a}}$ & $\geq 1$ & 93 & 19.5 & 45 & 14.3 & 15 & 14.6 & 153 & 17.1 & $X^{2}(2)=4.10, P=0.13$ \\
\hline Diagnostic procedures (inpatient/outpatient) ${ }^{\mathrm{b}}$ & $\geq 1$ & 222 & 46.5 & 145 & 46.2 & 48 & 46.6 & 415 & 46.4 & $X^{2}(2)=0.01, P=0.99$ \\
\hline
\end{tabular}

\begin{tabular}{|c|c|c|c|c|}
\hline \multirow{2}{*}{$\begin{array}{l}\text { Number of chest X- } \\
\text { rays }\end{array}$} & \multicolumn{3}{|c|}{ Number of chest CT scans } & \multirow[t]{2}{*}{ Total (column \%) } \\
\hline & 0 & 1 & 2 & \\
\hline 0 & $262(29 \%)$ & $148(17 \%)$ & $10(1 \%)$ & $420(47 \%)$ \\
\hline 1 & $94(11 \%)$ & $284(32 \%)$ & $16(2 \%)$ & 394 (44\%) \\
\hline$\geq 2$ & $7(1 \%)$ & $65(7 \%)$ & $8(1 \%)$ & $80(9 \%)$ \\
\hline Total (row \%) & $363(41 \%)$ & 497 (56\%) & $34(4 \%)$ & 894 (100\%) \\
\hline
\end{tabular}

admission, suggesting patients presenting with less severe or more typical lung cancer symptoms being referred to appropriate specialists. Nevertheless, fewer than half $(45 \%)$ of people attended a lung specialist (respiratory physician or cardiothoracic surgeon) in the lead-up to diagnosis, which is similar to an earlier NSW study that reported only $53 \%$ of people with lung cancer saw a respiratory physician at initial presentation. ${ }^{5}$ Reasons for not seeing a lung specialist in the community before a diagnosis of NSCLC could include the sudden onset of severe symptoms requiring emergency presentation to hospital (e.g. haemoptysis), referral to a non-lung specialist (e.g. an oncologist or a general physician), referral to a specialist for the presenting symptoms of distant metastases (e.g. a neurologist) or an incidental finding of lung cancer while undergoing investigation for another condition. An Australian study reported that $23 \%$ of people with lung cancer were diagnosed incidentally. ${ }^{7}$ Incidental findings will account for some people diagnosed without GP-ordered imaging or lung specialist attendance in our study.

There were no substantial differences in patterns of GP and specialist attendances and chest imaging for people living in outer regional and remote areas compared with major cities. It appears

\begin{tabular}{|c|c|c|c|c|c|c|}
\hline Rank & $\begin{array}{c}\text { GP- } \\
\text { ordered } \\
\text { imaging }\end{array}$ & $\begin{array}{c}\text { Lung } \\
\text { specialist } \\
\text { attendance }\end{array}$ & $\begin{array}{c}\text { Elective } \\
\text { admission }\end{array}$ & $\begin{array}{l}\text { Emergency } \\
\text { admission }\end{array}$ & $n$ & $\%(N=894)$ \\
\hline 1 & $\bullet$ & $\bullet$ & $\bullet$ & & 136 & 15.2 \\
\hline 2 & 0 & $\bullet$ & & & 132 & 14.8 \\
\hline 3 & & & & 0 & 129 & 14.4 \\
\hline 4 & - & & & & 119 & 13.3 \\
\hline 5 & & & & & 96 & 10.7 \\
\hline 6 & & $\bullet$ & & & 61 & 6.8 \\
\hline 7 & - & & & $\bullet$ & 61 & 6.8 \\
\hline 8 & & & 0 & & 45 & 5.0 \\
\hline 9 & & ○ & - & & 43 & 4.8 \\
\hline 10 & $\bullet$ & & 0 & & 39 & 4.4 \\
\hline 11 & - & • & & 0 & 18 & 2.0 \\
\hline 12 & & - & & 0 & 15 & 1.7 \\
\hline
\end{tabular}

that remoteness of residence alone is not a major barrier to seeing a lung specialist. However, we had no information on lung cancer MDT membership for the specialists, which may vary by remoteness. Further research is needed to determine whether referral to specialists who are active members of lung cancer MDTs is contributing to higher treatment use and improved outcomes for people diagnosed with lung cancer in major cities. $^{3,17}$

Overall, one quarter of people had an emergency admission for lung cancer in the lead-up to diagnosis. More than half of these 
did not have GP-ordered imaging or a lung specialist attendance. A higher proportion of people who had emergency hospitalisations had lung cancer with distant spread compared with those without an emergency admission, suggesting that symptoms of late-stage disease are leading to emergency presentations in some cases. Lung cancer symptoms requiring emergency treatment can occur with little or no prior warning or after a prolonged period without the patient seeking help for milder symptoms. ${ }^{18}$ While community awareness programs aim to increase recognition of symptoms and encourage people to seek medical advice, there is evidence that people delay seeking medical attention for reasons including a perceived lack of urgency about symptoms and stigma associated with lung pathologies if they have been smokers. ${ }^{19}$ Another reason for emergency presentation could be referral to an emergency department, either by a physician or patient self-referral, as a way to avoid real or perceived waiting time, financial or distance barriers to accessing a lung specialist or diagnostic procedures in the community. ${ }^{20,21}$

It is difficult to compare our pathway results with other studies because of differences in data sources, methods and health systems. The proportion of people diagnosed following an emergency presentation in this study $(25 \%)$ is lower than the figure of around one third reported in two other Australian studies and $39 \%$ in a UK study but much higher than the $6 \%$ in a Danish study. ${ }^{9,22-24}$ Although NSW emergency department data contains coded diagnostic information, these codes often reflect the presenting symptoms rather than the underlying diagnosis. The emergency department codes are assigned by a range of emergency department staff, whereas diagnoses in the NSW hospital data are coded by specialist medical coders using the medical record for each admission. Since people presenting to emergency departments who are diagnosed with lung cancer are likely to be admitted, we defined an emergency diagnosis pathway as one with an emergency hospital admission with a lung cancer diagnosis in order to avoid capturing people presenting to emergency departments who do not receive a definitive diagnosis. Another difference between studies is the definition of lung specialist. In the absence of information on lung cancer MDT membership, we included respiratory physicians and cardiothoracic surgeons, although some studies also include medical and radiation oncologists as lung specialists. ${ }^{4,5,7}$

A limitation of the data is that they do not contain presenting symptoms at GP and specialist attendances or imaging results. This meant that we were unable to calculate time intervals between first presentation with lung symptoms and diagnosis or treatment, assess the appropriateness of the observed pathways compared to a clinical guideline or standard, or identify people diagnosed incidentally. Although in our study health service use was similar across cities and rural areas in the lead-up to diagnosis, there may be disparity in the time between first presentation with symptoms and accessing specialist and diagnostic services that we could not measure. We were unable to identify a diagnostic procedure for around a third of people with a histopathological diagnosis, consistent with known under-reporting of diagnostic procedures in the hospital admission data. ${ }^{25}$

Smoking prevalence is lower among 45 and Up Study participants and smoking is the most important lifestyle risk factor in people presenting with lung cancer. ${ }^{19,26} 45$ and Up Study participants diagnosed with lung cancer were less socioeconomically disadvantaged, healthier and less likely to live in major cities than all NSW residents aged $\geq 45$ years diagnosed with lung cancer. ${ }^{27}$ Hospital and emergency department use in the year prior to lung cancer diagnosis were similar between 45 and Up Study participants and all NSW residents; however, primary and outpatient care use could not be assessed. ${ }^{27}$ Participants of cohort studies may differ from non-responders in their health literacy and health-seeking behaviours. This may result in differences in diagnostic pathways compared with the general NSW population with lung cancer.

Studies of diagnostic pathways are often based on patient medical record reviews, surveys or interviews and are restricted to small geographic areas or a limited number of hospitals. ${ }^{9-11} \mathrm{~A}$ strength of this study is that linkage of health-related records for 45 and Up Study participants enabled the examination of the use of primary care, outpatient imaging, specialist care and admissions to hospital in the lead-up to diagnosis of lung cancer for a NSWwide sample using data with population-level coverage, which had previously been a gap in understanding care pathways for lung cancer.

The Australian Optimal Care Pathway (endorsed in 2015) lays out expected pathways of initial investigations, referral, diagnosis and treatment for people presenting with suspected lung cancer with the aim of promoting best practice care regardless of where people live or have treatment. ${ }^{28}$ Our study focussed on GPordered imaging and specialist referral, which are steps laid out in the Optimal Care Pathway. The optimal pathway for a person with suspected lung cancer will depend on their presenting signs and symptoms and may include GP-referred imaging, referral to a specialist who is part of an MDT and referral of those with massive haemoptysis or stridor to the emergency department. Our study period is before the publication of the Optimal Care Pathway but can provide baseline data on health service use. Some areas in NSW have implemented localised optimal care pathways using GP decision aid software that provides management guidelines for people presenting with respiratory symptoms and referral details for lung specialists who are MDT members and for rapid access clinics. This implementation at a local level should assist with ensuring that people presenting with symptoms receive appropriate investigations in a timely way.

This study revealed that more than half of people did not attend a lung specialist in the diagnostic pathway of NSCLC. A quarter of people diagnosed presented as emergencies and more than half of those had no prior evidence of GP or lung specialist involvement in their diagnostic work-up. This study also highlights the key role that GPs have in the diagnosis of lung cancer. Ensuring that GPs have information available to them to initiate appropriate investigations and referral to specialists who are members of lung cancer MDTs has the potential to promote best practice care for people with lung cancer. Further research on barriers to recognition of lung cancer symptoms and access to lung specialists is needed from both a patient and physician perspective.

\section{METHODS}

Design and ethical considerations

This descriptive study used linked population-based, health-related data sets for participants of the 45 and Up Study. Ethical approval was from the NSW Population and Health Services Research Ethics Committee (HREC/14/ CIPHS/60). The 45 and Up Study was approved by the University of New South Wales Human Research Ethics Committee.

\section{Study cohort}

Our study cohort was selected from participants of the Sax Institute's 45 and Up Study. ${ }^{29}$ Participants were randomly sampled from the Department of Human Services (formerly Medicare Australia) enrolment database, which is a universal health-care scheme for citizens and permanent residents. Around 267,000 people joined the 45 and Up Study between February 2006 and December 2009 (18\% participation rate) by completing a questionnaire and providing signed informed consent to follow-up and linkage of health records.

Our analysis cohort included people diagnosed with NSCLC (see Supplementary Table 1 for definition) between enrolment into the 45 and Up Study and 31 December 2012. The focus is on NSCLC because it is the most common type of lung cancer and less common histology types can have different presentations and outcomes. People with another 
cancer case diagnosed between 2000 and 2012, a NSCLC notified by death certificate only or with an unknown date of diagnosis were excluded.

\section{Data sources}

Linkage of 45 and Up Study baseline questionnaire data to populationbased health-related data sets was used to identify cancer diagnoses and health service use. Cancer case data were obtained from the NSW Cancer Registry, a statutory registry of all invasive cancer cases (excluding nonmelanoma skin cancer) diagnosed in NSW residents. Hospital admission records for all NSW public and private hospitals were obtained from the NSW Admitted Patient Data Collection, which contains coded diagnostic and procedure information for each admission. GP attendances, specialist attendances, diagnostic imaging and diagnostic procedures performed in a community or outpatient setting were identified from Medicare Benefits Schedule claims data, which is a universal scheme providing subsidised medical services.

Linkage of the 45 and Up Study cohort to cancer and hospital data was performed by the Centre for Health Record Linkage (http://www.cherel.org. $\mathrm{au}$ ) and to the Medicare Benefits Schedule data by the Sax Institute using a unique identifier provided to the Department of Human Services.

\section{Cohort characteristics}

Demographic and cancer case data were obtained from the NSW Cancer Registry, including: date of diagnosis, most definitive method of diagnosis, extent of disease, age at diagnosis, sex, remoteness of residence (Accessibility/Remoteness Index of Australia [ARIA+]), and area-based socioeconomic disadvantage (Index of Relative Socioeconomic Disadvantage) ${ }^{30,31}$ Smoking status was obtained from the 45 and Up Study baseline questionnaire. The Charlson comorbidity score, excluding metastatic cancer, was calculated using hospital admissions in the 5 years prior to diagnosis. ${ }^{32}$

\section{Use of health services in the lead-up to diagnosis}

We quantified the use of GP attendances, specialist attendances, chest Xrays and chest $\mathrm{CT}$ scans in a community setting (outside a hospital admission), diagnostic procedures (bronchoscopy or biopsy) in an outpatient or inpatient setting and hospital admissions related to the diagnosis of lung cancer in the 15 months prior to diagnosis of NSCLC (see Supplementary Table 1 for definitions). We calculated the proportion of people using each service in the lead-up to diagnosis, which we defined as the 3 months ( 91 days) up to and including the date of diagnosis, based on the study data and the findings of other studies. ${ }^{12,21}$ The date of diagnosis on the NSWCR during the study period is the date of histological or cytological confirmation for the majority of cases or, for those with a clinical diagnosis, the date of first hospital admission or outpatient consultation.

\section{Pathways to diagnosis}

We defined pathways to diagnosis by the presence or absence in the leadup to diagnosis of:

- GP-ordered chest imaging (X-ray or CT scan);

- lung specialist (respiratory physician or cardiothoracic surgeon) attendance in a community/outpatient setting;

- hospital admission with a lung cancer indication, by urgency of first admission (planned or emergency).

These events indicate the involvement of GPs, lung specialists and hospitals in the diagnostic work-up. GP attendances were not included in the diagnosis pathway analyses because we could not distinguish lung cancer-related attendances from attendances for unrelated conditions.

\section{Statistical analysis}

We used Pearson's chi-square tests to assess associations between remoteness of residence and use of health services, and between outcomes of interest (seeing a lung specialist and emergency presentation) and patient characteristics or other events on the diagnostic pathway. Two-sided $P$ values are reported. Analyses were performed using SAS Enterprise Guide v6.1.

\section{DATA AVAILABILITY}

The raw data that support the findings of this study are available from the Sax Institute, NSW Ministry of Health and Cancer Institute NSW. Restrictions apply to the availability of these data, which were used under license for the current study, and so they are not publicly available. Data are, however, available from the custodians of the data sources by following the 45 and Up Study (https://www.saxinstitute.org.au/our-work/45-upstudy/for-researchers) and Centre for Health Record Linkage (www.cherel.org.au/applyfor-linked-data) application processes. Derived data are available from the authors upon reasonable request and with permission of the data custodians.

\section{ACKNOWLEDGEMENTS}

This research was completed using data collected through the 45 and Up Study (www.saxinstitute.org.au). The 45 and Up Study is managed by the Sax Institute in collaboration with major partner Cancer Council NSW and partners: the National Heart Foundation of Australia (NSW Division); NSW Ministry of Health; NSW Government Family \& Community Services - Ageing, Carers and the Disability Council NSW and the Australian Red Cross Blood Service. We thank the many thousands of people participating in the 45 and Up Study.

\section{AUTHOR CONTRIBUTIONS}

J.M.Y., D.B., N.C. and S.P. designed the study. S.P. performed the analysis. S.P. and N.C. drafted the manuscript. D.E., C.K.L., A.L., J.M., D.M., A.A.M., L.T., D.C. and K.M.W. provided comments on the design of the study and interpretation of the results. All authors reviewed drafts and contributed to the writing of the final manuscript.

\section{ADDITIONAL INFORMATION}

Supplementary information accompanies the paper on the npj Primary Care Respiratory Medicine website (https://doi.org/10.1038/s41533-018-0113-7).

Competing interests: The authors declare no competing interests.

Publisher's note: Springer Nature remains neutral with regard to jurisdictional claims in published maps and institutional affiliations.

\section{REFERENCES}

1. AlHW. Cancer in Australia 2017. Cancer series no.101. Cat. no. CAN100 (Australian Institute of Health and Welfare, Canberra, 2017).

2. Currow, D. C. et al. What factors are predictive of surgical resection and survival from localised non-small cell lung cancer? Med. J. Aust. 201, 475-480 (2014).

3. Tracey, E., McCaughan, B., Badgery-Parker, T., Young, J. \& Armstrong, B. K. Patients with localized non-small cell lung cancer miss out on curative surgery with distance from specialist care. ANZ J. Surg. 85, 658-663 (2015).

4. Simonella, L. et al. No improvement in lung cancer care: the management of lung cancer in 1996 and 2002 in New South Wales. Intern. Med. J. 39, 453-458 (2009).

5. Vinod, S. K. et al. Gaps in optimal care for lung cancer. J. Thorac. Oncol. 3, 871-879 (2008).

6. Vinod, S. K. et al. Underutilization of radiotherapy for lung cancer in New South Wales, Australia. Cancer 116, 686-694 (2010).

7. Mitchell, P. L. et al. Lung cancer in Victoria: are we making progress? Med. J. Aust. 199, 674-679 (2013).

8. Evans, S. M. et al. Timeliness of lung cancer care in Victoria: a retrospective cohort study. Med. J. Aust. 204, 75.e1-75.e9 (2016).

9. Largey, G., Ristevski, E., Chambers, H., Davis, H. \& Briggs, P. Lung cancer interval times from point of referral to the acute health sector to the start of first treatment. Aust. Health Rev. 40, 649-654 (2016).

10. Emery, J. D. et al. Diagnosing cancer in the bush: a mixed methods study of GP and specialist diagnostic intervals in rural Western Australia. Fam. Pract. 30, 541-550 (2013).

11. Lacey, K. et al. Presentations to general practice before a cancer diagnosis in Victoria: a cross-sectional survey. Med. J. Aust. 205, 66-71 (2016).

12. Hamilton, W., Peters, T. J., Round, A. \& Sharp, D. What are the clinical features of lung cancer before the diagnosis is made? A population based case-control study. Thorax 60, 1059-1065 (2005).

13. Walter, F. M. et al. Symptoms and other factors associated with time to diagnosis and stage of lung cancer: a prospective cohort study. Br. J. Cancer 112(Suppl 1), S6-S13 (2015).

14. Cancer Australia. Investigating symptoms of lung cancer: a guide for GPs. https:// canceraustralia.gov.au/publications-and-resources/cancer-australia-publications/ investigating-symptoms-lung-cancer-guide-gps (2012). 
15. Prabhakar, C. N., Fong, K. M., Peake, M. D., Lam, D. C. \& Barnes, D. J. The effectiveness of lung cancer MDT and the role of respiratory physicians. Respirology 20, 884-888 (2015).

16. Boxer, M. M., Duggan, K. J., Descallar, J. \& Vinod, S. K. Do patients discussed at a lung cancer multidisciplinary team meeting receive guideline-recommended treatment? Asia Pac. J. Clin. Oncol. 12, 52-60 (2016).

17. Chen, T. Y. et al. Survival from breast, colon, lung, ovarian and rectal cancer by geographical remoteness in New South Wales, Australia, 2000-2008. Aust. J. Rural. Health 23, 49-56 (2015).

18. Abel, G. A. et al. Emergency diagnosis of cancer and previous general practice consultations: insights from linked patient survey data. Br. J. Gen. Pract. 67, e377-e387 (2017).

19. Crane, $M$. et al. Knowledge of the signs and symptoms and risk factors of lung cancer in Australia: mixed methods study. BMC Public Health 16, 508 (2016).

20. Australian Bureau of Statistics. 4839.0 - Patient Experiences in Australia: Summary of Findings, 2012-2013 (Australian Bureau of Statistics, Belconnen, ACT, 2013).

21. Hall, S. E. et al. Lung cancer: an exploration of patient and general practitioner perspectives on the realities of care in rural Western Australia. Aust. J. Rural Health 16, 355-362 (2008).

22. Yap, S. et al. Patterns of care and emergency presentations for people with nonsmall cell lung cancer in New South Wales, Australia: a population-based study. Lung Cancer 122, 171-179 (2018).

23. Elliss-Brookes, L. et al. Routes to diagnosis for cancer - determining the patient journey using multiple routine data sets. Br. J. Cancer 107, 1220-1226 (2012).

24. Guldbrandt, L. M., Fenger-Gron, M., Rasmussen, T. R., Jensen, H. \& Vedsted, P. The role of general practice in routes to diagnosis of lung cancer in Denmark: a population-based study of general practice involvement, diagnostic activity and diagnostic intervals. BMC Health Serv. Res. 15, 21 (2015).

25. Goldsbury, D. E., Armstrong, K., Simonella, L., Armstrong, B. K. \& O'Connell, D. L. Using administrative health data to describe colorectal and lung cancer care in New South Wales, Australia: a validation study. BMC Health Serv. Res 12, 387 (2012).

26. Mealing, N. M. et al. Investigation of relative risk estimates from studies of the same population with contrasting response rates and designs. BMC Med. Res. Methodol. 10, 26 (2010).
27. Creighton, N. et al. Representativeness of health service utilisation among participants of a population-based cohort study diagnosed with cancer compared with the source population: a data linkage study. BMC Med. Res. Methodol. 18, 84 (2018).

28. Optimal care pathway for people with lung cancer. http://www.cancer.org.au/ content/ocp/health/optimal-care-pathway-for-people-with-lung-cancer-june2016.pdf (2015).

29. 45 and Up Study Collaborators et al. Cohort profile: the 45 and Up Study. Int. J. Epidemiol. 37, 941-947 (2008).

30. Australian Bureau of Statistics. 1216.0.15.003 - Australian Standard Geographical Classification (ASGC) Remoteness Area Correspondences (Australian Bureau of Statistics, Belconnen, ACT, 2011).

31. Australian Bureau of Statistics. 2039.0 - Information Paper: An Introduction to Socio-Economic Indexes for Areas (SEIFA), Australia, 2006 (Australian Bureau of Statistics, Belconnen, ACT, 2008).

32. Quan, H. et al. Updating and validating the Charlson comorbidity index and score for risk adjustment in hospital discharge abstracts using data from 6 countries. Am. J. Epidemiol. 173, 676-682 (2011).

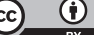

Open Access This article is licensed under a Creative Commons Attribution 4.0 International License, which permits use, sharing, adaptation, distribution and reproduction in any medium or format, as long as you give appropriate credit to the original author(s) and the source, provide a link to the Creative Commons license, and indicate if changes were made. The images or other third party material in this article are included in the article's Creative Commons license, unless indicated otherwise in a credit line to the material. If material is not included in the article's Creative Commons license and your intended use is not permitted by statutory regulation or exceeds the permitted use, you will need to obtain permission directly from the copyright holder. To view a copy of this license, visit http://creativecommons. org/licenses/by/4.0/.

(c) Crown 2019 